\title{
Roles and Responsibilities of Family Home Caregivers for Elderly People in Qatar: Experiences and Challenges
}

\author{
Azza O Abdelmoneium ${ }^{1}$, Janet Rankin², Michael Corman ${ }^{3}$ \\ ${ }^{1}$ Lead Researcher, Doha International Family Institute/ Qatar Foundation, PO box 34080, Doha, Qatar \\ ${ }^{2}$ Associate Professor, University of Calgary, Qatar \\ ${ }^{3}$ Adjunct Professor, Department of Sociology and Anthropology, The University of Prince Edward Island, Canada \\ Correspondence: Azza O Abdelmoneium, Lead Researcher, Doha International Family Institute/ Qatar Foundation, PO \\ box 92745 , Doha, Qatar.
}

Received: May 12, 2017

doi:10.11114/ijsss.v5i7.2413
Accepted: May 31, $2017 \quad$ Available online: June 7, 2017

URL: https://doi.org/10.11114/ijsss.v5i7.2413

\begin{abstract}
The family, as a social institution in Qatar, is the main care provider for its older adult members. In this paper we examine what caregivers do - their work practices - and the different roles and responsibilities that home caregivers perform when providing care for elderly persons in Qatar. The findings of this study were based from a larger study using qualitative descriptive design. This article demonstrates the work practices and main challenges that caregivers face while taking care of their elderly. We argue that a main source of stress for caregivers is the lack of formal services and supports geared towards individuals caring for their ageing loved ones; addressing this service gap would alleviate some of the demands associated with caregiving. The paper will present exemplars of caregivers taking care of their elderly relative. These exemplars are used to support the analysis which is clustered around identified related themes. The main findings regarding the challenges that caregivers face include: feelings of responsibility; dependency on caregiver; emotional care; social planning; problem solving; and environmental quality. All of the themes impacted the health of the caregivers and led to stress and burden on the life of the caregiver.
\end{abstract}

Keywords: family home caregivers, family, elderly people, roles, responsibilities

\section{Introduction}

The family is an important support for older individuals, especially when the older person's physical and/or mental health declines and when they can no longer function independently whereupon it is often members of the family who become informal caregivers for the elderly person or persons requiring support. Internationally, there is an abundance of social research on the experiences of caregivers for individuals of advanced age. Howe ver, there has been no research conducted in Qatar and only a few studies related to elderly people in the Arab region (Economic Social Commission for Western Asia, 2013). This research gap is problematic for two reasons. First, Qatar has seen significant increases in life expectancy since the 1950s due to a variety of socio-economic factors (Qatar Statistics Authority, 2010), resulting in not only the number of people aged 25-64 years increasing substantially but also an increase in the number of adults over the age of 60. In 2010 the number of Qataris and non-Qataris above the age of 60 was estimated to be 10,756 and 18,030 respectively (Qatar Statistics Authority, 2010). That number is expected to increase significantly; with an average life expectancy extending from 78 years of age to 80 years of age in Qatar by 2020 (United Nations, 2012). In fact, Qatar is one of the fastest aging countries in the Middle East (Saxena, 2008). Second, a recent report suggest that there are problems (such as neglect, abuse, violence and lack of support for caregivers) in older persons' care in the Arabic world (ESCWA, 2013).

The findings from this paper are based on a larger study that qualitatively explored the experiences of family caregivers in Qatar who look after elderly relatives at the home. This article specifically focuses on the roles and responsibilities that home caregivers perform for their elderly people and the challenges they face in practicing this task. Based on the findings, we argue that a coordinated, well-integrated system of formal assistance (current home health services are fragmented across primary and tertiary health organizations and small community organizations) would improve 
services for elderly care in the home. With better supports, home caregivers might experience less stress and burden from the caregiving process and enjoy a higher quality of life with the care receiver.

\section{Background}

The Work and Family Researchers' Network define family caregiving as:

The act of providing unpaid assistance and support to family members or acquaintances that have physical, psychological, or developmental needs. Caring for others generally takes on three forms: instrumental, emotional, and informational caring. Instrumental help includes activities such as shopping for someone who is disabled or cleaning for an elderly parent. Caregiving also involves a great deal of emotional support, which may include listening, counseling, and companionship.... (Work Family Research Network, 2010).

Family is not the only people who provide homecare for old persons. In the published research caregivers are categorized as professional and personal caregivers. Professional caregivers work for pay and are generally part of the skilled formal health care sector such as nurses, physical therapists, occupational therapists, speech therapists or social workers. Personal care or informal caregivers are usually family members or non-professional workers at home. In regards to care provided in the home, informal caregivers have been found to be motivated by a deeper commitment to the elderly person than the professional group of carers (Grunfeld \& Ian, 1997:1101). Research shows that most homes are organized around a structurally based gender division of labour whereby women take the responsibility of primary caregivers and homemakers.

The large body of international literature that discusses common trends in a gendered division of labor for caregivers include Lin and Hsueh-Sheng (2012); Neal, Berit and Marjorie, (1997); Piercy (1998); Singleton (2000); Stein (1998); Teschendorf and Carolyn (2007) and Walker, Clara, Pratt and Lindy (1995). The studies that identify the gendered division of caregiving show that, while there may be an extended network of carers, it is often a woman who is the primary caregiver. The primary caregiver is involved directly with Activities of Daily Living (ADL) (Messecar, 2012; Capezuti, Deanne, Mathy, \& Terry, 2008). These include day-to-day assistance such as bathing, eating, dressing, mobility, transferring from one place to another, and using the toilet. Illness related care extends beyond the basic bodily work and includes carrying out treatments, performing medical or nursing procedures, coping with illness behaviors such as cognitive impairments and, liaising with health professionals and navigating the health care and social services systems. Many of these are protective actions that caregivers take to ensure the older person's safety and wellbeing (Bowers, 1987; Schumacher, Barbara, Patricia, Mildred, Faith \& Sangeeta, 2000; Smith, 1994). Other Instrumental Activities of Daily Living (IADL) such as meal preparation, shopping, grocery, housework, making phone calls, income generation and money management contribute to the wellbeing of an elderly person being cared for in the home and the contribution of IADL cannot be underestimated.

In the literature, the roles of the caregiver have been classified according to who takes the major responsibilities (primary caregiver) versus those people who are categorized as having only supportive roles (secondary caregivers). Secondary caregiving has some similarities to the work of the primary caregiver. However, often the day-to-day contributions are intermittent because the secondary caregivers are involved in full-time employment or they live at a distance from the home of the dependent elder (Philips, 1989). According to Swanson, Deborah, Janet, Marion and Meridean, (1997) and more recently Lou, Shuling, Ya,, Mengyuan, Shuai and Yong, (2015) the roles and responsibilities of the secondary caregiver are often critical to the well-being of the dependent elder. In some cases the role of the secondary caregiver is to provide respite for the primary caregiver. In this regard, the secondary caregivers may assist with the ADL and IADL. In the research being reported here we analyzed the role of the primary caregivers.

According to Messecar and others, female spouses are most likely to assume the primary caregiving role, whereas adult children are more likely to be secondary caregivers (Messecar 2008:130; Schoenfelder, Swanson, Pringle, Meridean \& Johnson, 2000) with daughters implicated in caregiving more than sons. Schoenfelder et al, (2000) closely examined the gendered responsibilities and identified that it is the wives, daughters, and daughters-in-law who provide the bulk of the personal care for the elderly family member. In Schoenfelder's study, husbands and sons were noted to be monitoring the care of elderly relatives from a distance, most often living separately from the ageing relative, they were seen to be intervening and offering advice or directions by telephone or during visits (see also Dwyer \& Coward, 1991).

Many of the primary caregivers in our study did not have a spousal relationship with the relative they were providing care for and we found both adult sons and daughters involved as primary caregivers. The tradition of family caregiving is essential in Qatar (Grunfeld \& Ian, 1997:1101) and in our small group of participants there was a predominance of women who identified as primary caregivers. Sixty three percent of the caregivers we interviewed were single wo men (daughters who either never married or divorced) who provided most of the care for their parents. While our qualitative study cannot make broad claims about gender, our findings suggest that, in Qatar, this gendered division of labor is likely consistent with prior research. 


\section{Methodology}

The interviews were conducted in 2015 in Doha, the capital of Qatar. Qatar. The research employed a descriptive qualitative description research design with "hues" of phenomenology (Sandelowski, 2000). This design was geared towards gaining an understanding of the experiences of family caregivers. In the context of this study, the research goal was to respectfully open a view into the private realm of family life in order to gain insight into the complicated nature of caregivers' lived experiences and to identify how their family caregiving role could be supported and enhanced. Aligned with qualitative research, this study used an inductive approach to generating knowledge through in-depth interviews which were used to identify patterns based on the experiences of participants (Rudestam \& Newton, 2001).

We recruited 22 people using posters and word of mouth at the organizations where the three researchers worked. This small convenience sample revealed serious challenges for all of the respondents in practicing their roles and responsibilities towards taking care of their elderly people. The inclusion criteria for the 22 participants was "Qatari and non-Qatari caregivers residing in Qatar, who self-identify as full time or part-time caregivers and who are caring for family member aged $65+"$

Among the 22 participants, five were male and 17 were females. Out of the 17 females there were ten Qatari Nationals and seven non-Qatari long term residents. The five males were all non-Qatari Nationals who were also long term residents. The age range of the caregivers was between 20-50 years old. Most caregivers considered themselves the primary caregiver, although discerning between the roles of primary or secondary caregiving was sometimes difficult because the caregiving work was organized within a complex sharing of responsibilities. Out of the 22 participants, 11 held employment (or were attending post-secondary education) either full or part-time.

\section{The Lived Experience of Being a Primary Caregiver- Case Studies}

In our study, caregiving for older family members was mostly carried out by relatives and took many forms. In this paper, we focus on the different types of work processes described by caregivers associated with their caregiving roles. The process of caregiving for the elderly persons is a complex issue. For instance, caregivers are often required to gain specialized knowledge unique to the needs of the individual they are caring for. Regular appointments with healthc are professionals are an important component of monitoring ongoing health decline and developing nuanced caregiver expertise during the different stages of the caregiving process (Given, Sherwoon \& Given, 2008; Given, Given \& Kozachik, 2001; Northouse, Mood, Templin, Mellon, \& George, 2000).

In this section we highlight the experiences of two participants explaining their roles and responsibilities towards their elderly relative. Noor, is a single Qatari woman in her thirties who is employed full-time outside the home and is also responsible for her father and mother (both aged in their 60s). Noor's father has several chronic illnesses that have led to limited vision. Her mother has diabetes, hypertension, and other health issues, she also has a complex chronic wound which is being treated at home. Noor reported that she had to negotiate with her employer to get permission to attend medical appointments with her father or mother. Although she has brothers and sisters, all but her youngest brother, in his 20s, are married and live in their own homes. The brother lives with Noor and her parents but he is not closely involved in the day-to-day care. Noor described her daily routine:

I wake up every morning, I prepare their breakfast. My mother wakes up at five. I give breakfast for my father and mother, I give them their medication and then I go to work. I tell the housemaid to give them juice at 11 and check their sugar level. At noon when I come back I check that they had their medications and I prepare the medications for the rest of the day or the second day. When I come back from work at $3 \mathrm{pm}$ I prepare their lunch and then I sit with them for the rest of the day and give them their medications and any other issues that they need. Every two days we need to check the foot (her mother's wound) and clean it. Sometimes the housemaid does it but normally I do it. I wash their feet I check what is missing in the house in terms of food or other things that needs to be done.

She went on to explain the following:

The housemaid helps me in the cleaning of the house and looking after my parents when I am at work, but sometimes when you do not have a good housemaid you get scared and I tend to leave work and take permissions to go back home and look after my parents, because no one will look after them. My younger single brother is working and my other sisters and brothers all have their own lives and responsibilities. So I have a hard time when I do not have a good housemaid as everything will be on me.

Noor's experience matches with what has been written in the literature in-so-far as she fits the international demographic of women who function in the caregiving role for an elderly parent in need of assistance (see Brody 1990; Cantor, 1983; Singleton, 2000). Noor describes that she takes care of her parents' practical needs, while her other 
siblings are involved in the social aspects of her ageing parents' wellbeing. Despite that Noor has hired a housemaid, it became clear through speaking to Noor that she bears the responsibility of the day-to-day worries and negotiating her parents' medications, their need for regular snacks and the support they need related to their disabilities and conditions. Noor finds it difficult to trust the housemaids with any of the primary responsibilities in the care of her parents.

May is a Qatari single female in her 50s who is employed part-time and takes care of her 70 year old mother who suffers from breast cancer and has a complex health history. For the past four years, May has been fully responsible for her mother and although she has a housemaid she reports that she maintains responsibility for all the housework. She has siblings. Some brothers who are unmarried live at home. Her sisters are all married and live outside of the home, May considers herself the primary caregiver. May describes her mother's health challenges and the care that her mother requires as follows:

Since the past seven years my mother has undergone four major operations ... All these operations made my mother frail and sick needing assistance from me. I take care of my mother because my sisters are all married and after each operation she needs time to recover and rehabilitate so all the duration that she stays in the hospital I am the one who accompanies her. I am 100\% responsible of all the shopping, cooking, managing medications, doing housework, managing finances and payments of bills. Other personal activities that I assist my mother [with] are: bathing, dressing, toileting, walking, eating, and transferring beds, particularly at the time after the operation. My mother was completely dependent on me on these issues after the operations that she did, but after she recovered she manages by herself but needs supervision and assistance. I sleep with her in the same room and assist her in everything that she needs or wants. Sometimes she asks to buy things from the shop. I go and buy it for her. I cannot refuse to say no because she can be offended. I try as much as possible not to make her sad, I always delay my personal things for the sake of doing what she is requesting.... I try to be sensitive with her issues and obey what she wants so as not to feel that she is incapable of doing what she used to do before the operations. For example I put her to sit in the kitchen and supervise what the housemaid is doing and tell the housemaid how to do things. This thing makes her feel included in daily activity and does not feel bored.... She sometimes tells the driver that she wants things from the shop to buy for her so she tells the driver the way and from where to get them. Things like that make her think and feel included and not isolated... . We always have lunch with one of my younger brothers staying with us in the house. She feels happy that we have lunch together.

The descriptions that Noor and May provided are typical of the primary role in caregiving work and the secondary contributions of their siblings are similar. The work of caregiving is complex and includes multiple work processes. The role of the primary caregiver and the time and effort exerted in the caregiving process has practical, social and emotional challenges on the primary caregiver which are discussed below.

\section{Secondary Caregivers}

Most of the primary caregivers interviewed reported getting assistance from other family members. Some received regular assistance from their siblings who are living away from them. This was often in the form of regular visits when the siblings joined the primary caregiver for evening meals or family social time.

Another form of secondary caregivers was the foreign domestic housemaid. Most of the individuals we interviewed had a foreign domestic housemaid. Some of the participants said that the housemaid does the cleaning and food preparation tasks, while others spoke about how the housemaid was central to their well-being and the well-being of the receiver. Some housemaid was more connected to the physical care of the elderly person helping with lifting, dressing or bathing. Most of the cases emphasized that medications were not given by the housemaid. In this paper we will focus on the challenges of the primary caregiver.

\section{Chal lenges of being a Primary Family Caregiver in Qatar}

In this section, we describe some of the challenges of being a primary caregiver that our data revealed. We discovered the burdens and feelings of responsibility incurred within the older relative's dependency, and the ongoing sense of worry that caregivers described. They worry not only about practical issues of physical care but also their relative's emotional care where they talked about inclusion and being sensitive to the elderly person's dignity and status in the family. We also describe how the caregiving role results in social challenges that require planning and problem solving.

\subsection{Feelings of Responsibility}

Fears about leaving the dependent elder were expressed by several of our study participants. There seemed to be an overlying specter of worry if the primary caregiver had to leave the house. All of the primary caregivers developed complex systems to address the worry. It seems that the mobile phone is indispensible for communication among the 
primary and secondary caregivers and the mobile phone seemed to facilitate primary caregivers' capacity to carry out activities outside of the home. This was especially the case for the participants who left the home for employment or to attend university.

All the caregivers expressed a pervasive high level of worry and concern for their elderly family member. This was accompanied by expressions of strong feelings of personal responsibility. For some, this extended to ongoing worries about "something happening" and their expressed sense of guilt and failure if they were to be away when they were needed. Even when other family members lived in the house and had close knowledge of the complexity of the caregiving work, the primary caregivers seemed to bear primary worry. They told us the other family members did not seem burdened by the weight of responsibility, nor the worries and tensions that the primary caregivers experienced. There were gendered elements to this role of responsibility in regards to sons (the caregivers' brothers) who lived in the same house but did not seem to bear the burden of worry that their sisters experienced.

\subsection{Dependency on the Caregiver}

Another challenge related to this sense of worry is the level of dependency of the elderly person. This was the case for study participant Mo who was the primary caregiver for his father who depended on him for every trip to the toilet and whose sense of responsibility and worry was greatly magnified. Even when the elderly parents were physically more able the sense of responsibility for such issues as good diabetes management and the risk of diabetic emergencies such as hypoglycemic coma established critical dependency on the primary caregiver. In all cases, the caregivers "provided assistance above and beyond aid given to physically and psychologically healthy members. Care giving is provided to someone who is dependent on that assistance" (Walker et al 1995:403). All of the elderly people the study participants provided care for were dependent on the assistance they received from their caregiver.

The elderly were dependent for shopping, for transportation to and from medical appointments. They were dependent on caregivers for social inclusion that supported them to feel loved and wanted - not isolated. These sorts of dependencies accompanied the other features of IADL and ADL activities. Although we did not measure the amount of time the caregivers spent in providing care, the descriptions revealed that the caregiving role absorbed a great deal of time each day. Even when the caregiver was not directly absorbed in IADL or ADL, there was a mental energy that linked the caregivers to the care receivers.

\subsection{Emotional Care}

The data revealed that caregivers negotiate a variety of ways to provide for their relatives' emotional needs which presented as a challenge to some of the caregivers. The caregivers described how providing ongoing emotional support in the face of caregiver fatigue and the high expectations of the care receiver is not an easy emotional terrain to balance. All of the caregivers in our study demonstrated the ways they generate patience, respect, and dignity. Noor talked about how she returns home from work and then spends the afternoon and evening with her mother, trying to "make up" for the isolation she knows her mother experiences when she is not there. She described the day-to-day irritations that she and her mother experience together but she emphasized how she sees it as her job, when she comes home from her paid work, to always obey her mother and consistently provide what she wants. Participants Dream and Mony revealed how they prioritized time with their fathers in order to give them emotional support and comfort; their attention was consistently focused on reducing their fathers' sense of loneliness or depression. These types of emotional exchanges influenced the relationship between the caregiver and care receiver and seemed to contribute to overall improvements in quality of life. Even in the face of irritation, frustration and annoyance that is inevitably a part of the caregiving experience, the caregivers maintained their value for being patient and providing an emotional climate that demonstrated care and dignity.

These findings are supported by research that has shown that socio emotional care is important to the caregiving context and is closely related to the instrumental aid given to dependent family members (Walker et al 1995: 404). A strong theme in most of the interviews we analyzed was the attention to emotions and thoughtful interactions with the older person. In fact, some participants emphasized that emotional care is as important as medications, eating, and hygiene. This finding related to the importance the caregivers placed on emotional support can be identified as a core component to the quality of care that the dependent elderly relatives of the study participants received from their family caregivers. Research shows that ignoring emotional care has an impact on the health of the care receiver and can lead to health deterioration (Dahlberg \&McKee, 2014; Hawkley \& Cacioppo, J.T., 2010; Walker et al 1995). Research has also shown that failure to consider people's emotional moods in the caregiving process is a component of "decontextualized caregiving" (Walker et al 1995:404) whereby the elderly person is not addressed in the context of a long and complex life, but merely seen as they are now, frail and dependent (see also Milligan, 2005). Attending to emotional wellbeing of their elderly relative is an important finding in this preliminary descriptive study. It suggests that the current norm in Qatar that positions the family as the main social institution for elderly care is an important value to promote and 
support. It is an important differentiation between elderly care in other cultures where the cultural norm accepts "care homes."

\subsection{Emotional Energy Directed towards Inclusion}

Within the care giving roles and responsibilities, an important issue related to emotional care focused on the inclusion of the elderly person in family life. All the participants expressed that they try their best to include their elderly person in their lives in a variety of ways regardless of the level of the older person's functional impairment. This goal of the caregivers had nuanced differences from how they discussed emotional support. The caregiving efforts directed towards inclusion were focused on ensuring that the elderly relative felt needed and knew they were a valuable contributor to family life. Bless, for example, explained how she goes "out with my father for walks and on Fridays the rest of my siblings join us with their children and my father gets happy about that."

Abu Ali also engages with his father. Abu Ali works full time, comes home late and does not find time to stay long with his father. He said

I feel he get upset when I tell him that I am going to sleep or see check on my children, so I try to not upset him by laughing or staying for some time and leave him... I bring my children to play with him and entertain...

Inclusion of the old person within the family increases the quality of life and sustains health; the more an elderly person is involved in the family, the more s/he feels integrated and this appears to have positive health benefits (Piercy 1998; Butcher \& Buckwalter, 2002). This was made visible by Blue when he explained that including his father in family life makes him "happy." He said

In the afternoon time we take our father to sit in the garden and all the rest of our siblings or friends come and visit us to chat with him. This entertains him and makes him happy. I keep talking to him about our family and news in the world to engage in conversation and discussion with me. Sometimes it is not easy to keep talking but we do our best.

\subsection{Emotional Energy and Responding Sensitively Challenge}

Being sensitive to the needs and desires of older family members is a factor that the caregivers believed was an important part of their caregiving role. Some of the caregivers likened their elderly relatives to the younger members of the family; they felt that like children, who are tender, fragile and vulnerable, older people need similar support, affection and care. However, they were also sensitive to the wisdom and autonomy that comes with age and were sensitive to issues of autonomy and control. They tried to make sure that the household affairs were maintained in a similar way to how their elderly parents "ran things" prior to becoming dependent.

Um Ibrahim, a single Qatari woman in her 40s who was the primary caretaker for her ageing mother, described how "my mother sometimes likes to cook alone although we have two housemaid, but my mother is the one who supervises the cooking, this makes her busy during the day". In this regard, Um Ibrahim was respectful of her mother's many years of culinary expertise and created a safe environment for her mother to continue to be active in the kitchen. Jamie, also a single woman, said: "I love my father very much and since we are a big family and we have kids, every time my father comes from outside I make sure that his room is clean before he enters. I really care for this. Sometimes there are lots of shoes, most of the things the shoes outside. I put e verything in order just to make him happy". These descriptions reflect that the caregivers cared about sensitive issues that might disturb the elderly person. Jamie cares that her father's room is clean and tidy. She understands this makes him happy as she knows how much he likes a clean place.

Sensitivity also was encountered by respecting to the wishes of the older persons. Karkar, a single Qatari woman in her 40s taking care of her mother and grandmother mentioned:

Me and my siblings try to arrange programs for both my mother and grandmother. For my grandmother she likes to go to the farm so we take her twice a month. She also likes fishing so we arranged for her a boat as she likes going to the sea a lot.

Mony, a 25 year old female taking care of her father disclosed how she preserves her father's autonomy by doing what he asks her to do. Things that her father would have normally done, he directs Mony to do them even though they are jobs that, before his decline, he would not have considered "proper" for a woman. Mony said:

Sometimes my father asks me to do things that are hard for me. Mainly for men, such as moving a table to the other, fixing stuff like giving me a hammer to fix things and I do it for him because he asked me.

This acquiesce to her father's directions and her efforts to "fix" things reveal a deep sensitivity to her father's pride and 
how difficult it would be for him to ask an outsider to come and do the small home repairs that Mony undertakes with his direction.

Being sensitive and considerate to the requests of the old person is important in the caregiving process and generates mutual relationships.

Sometimes responding sensitively means being highly attuned to predicting the demands upon and energy reserves of the older person. For example Hope, a female caregiver taking care of her mother said:

Waiting in the clinic is unbearable for my mother. She needs to go to the bathroom frequently and she takes diuretic pills so on the day we are going to the clinic I stop the pills but stopping the pills make her feet swell and that is not good for her. But I have no choice.

Hope is closely tuned in with her mother's medication regime and its effects on day to day life. She uses this intimate, personal knowledge with skilled sensitivity working to carefully balance her mother's comfort.

All the people interviewed expressed a desire to respect the wishes of their elderly relative. They expressed fulfillment in their caregiving role when the elderly relative was integrated and not isolated and lonely. Achieving this integration became more difficult with increasing cognitive or physical debility but all the caregivers described their ongoing efforts to engage their elderly relative as much as possible and support them to participate in the daily activities of home life. By doing so, the caregivers believed that the elderly person would experience less frustration and their quality of life will be enriched, despite how much stress and tension is exerted.

\subsection{Social Planning and Problem Solving Challenge}

Extending from the sensitive emotional care that the caregivers provide within a demanding set of circumstances, the caregivers actively developed action plans and embarked in problem solving. Sometimes this creates feelings of anxiety. For example, Hope's ad hoc decision to not administer the diuretic (water pill) on the morning when there is a medical appointment reflects her sensitivity to the anxiety that is created when her mother experiences the need to urinate when waiting for a doctor's appointment. However, this sort of planning and decision making can be more stressful because they require Hope to make medical decisions that she feels unqualified for. She expressed misgivings related to the limited options available to her when she is sensitively working to support her mother's wellbeing. Noor, a woman who is employed outside the home and is also primary caregiver of her parents, had an experience similar to Hope's. She said "My mother is diabetic and needs care and cleaning her foot because of fungus several times a week. I work and sometimes I get tired so I trained the housemaid to clean it for her".

Caregivers need to plan ahead and or make a plan of action and follow it to cope with complex circumst ances in the caregiving process. To plan action or to solve a problem helps reduce the cause of the stressor. Mo, who is a male student taking care of his father said: "I feel stressed when the exams are near but I arrange with my siblings to take care of my father". Hope said: "If I am travelling outside Qatar I ask my sisters to come and take care of my mother". Noor said:

I work full time and if I am late at work, I ask the house maid to give my parents their food and juice but not medications. I usually prepare their food before I leave to work so I plan ahead.

Some caregivers seek the help of others within the family who can help solve their problems when they encounter a stressful event. As Dream said: "I ask my sister to help me when I hospital my father, I stay at night with him but in the morning my sister or brother take over and stay with him in the hospital".

The caregivers in this paper managed to solve their stressful events by either planning ahead to avoid stress or devising responsive solutions to the problem as in the case of Green.

\subsection{Time Management Challenge}

Another challenge that the caregivers respond to is effective time management. Time management is difficult to some caregivers because they need to manage their time to accommodate their own occupations as well as accommodating the needs of their dependent relative. Most of the caregivers in this study complained about the long hours of waiting in the clinic/hospital. Hope emphasized this and said:

When we have an appointment for my mother in the hospital we have to leave the house three hours before the appointment, because of traffic jam and finding a parking lot. Although there are parking lots for disabled but always occupied. By leaving early we arrive on time but we have to wait in the waiting room till we see the doctor.

Mony, a young woman who is a student said:

During my examination time I double my effort and study at night after my father sleeps to finish my 
studies... because during the day it is not easy to find time, this cause me stress but I solve it by studying at night.

It is stressful indeed for the caregivers when they are working or studying at the same time they are primary caregivers. They need the support from other members within their families, but sometimes members within the family are not cooperative or available to them.

\subsection{Environmental Quality Challenge}

The caregivers we interviewed cared about the quality of the environment of their elderly relatives. The concern for the environment was not only comfort and aesthetics but also protection and security. The caregivers assumed the responsibility for the quality of the environment although the financial resources for providing a comfortable, safe, environment were often shared.

In particular, the elderly person's safety was carefully considered and worried about. For example, Bless, a woman and Qatari national in her 40's had been taking care of her blind, father in his 70's for several years. She worries about her father's safety as he adapts to his vision loss. He has now been totally blind for two years. She also manages the medical care of his diabetes and hypertension. For Bless to maintain a comfortable and protective environment in the house she prepared a room that reflected his unique needs:

In the bathroom we have a special bathroom chair in the bath for my father to sit on. I arranged for him in the outside a ramp for his wheel chair to be used. The downstairs room that I am preparing for him has all things to use for a disabled person such as chair, bed, and exercise machine. It is still under preparation but will finish soon. Me or the housemaid do for him massage, nail treatment, hair dressing. He likes to hear the news so I always open the TV on news channel in a loud voice to let him hear it and sometimes if there is important news that he missed I tell him. I try to make things easy for him to access in the house and to know his whereabouts. This is important because my father used to be an independent person and now after he lost his sight I do not want him to feel that he lost his independency. I try to make everything in the house comfortable for him.

The situation is quite similar to Blue, a man in his 30's who for eight years has been taking care of his father paralyzed by a stroke who explained how:

Since my father got paralyzed we provided for him a comfortable medical bed with a medical mattress that assist him in sleeping and avoid any bed injuries. We also have a wheel chair for him and we arranged his bathroom with all handles for a disabled person.

All of the participants in this study initiated a discussion about the safety and security of the old person. Preventing falls and avoiding medical complications were a key focus of caregivers' concern. The caregivers expressed that the environment must be a comfortable and safe environment. They also noted that their caregiving work was easier when they had the right equipment and the house was laid out in a way that was conducive to physical care. For example Blue indicated that they purposefully chose a 3-bedroom apartment that had no stairs in order to make it easier to care for his father. In Bless' case, the "downstairs room" being prepared meant that her father would no longer have to navigate a staircase to go to bed. It was apparent that the more resources there were to produce a safe and comfortable environment, the easier it became for caregivers to provide the needed physical, emotional, and environmental care for their dependent elderly relative.

\subsection{Financial Burden}

The caregivers we interviewed discussed the financial burden that they experienced while taking care of their elderly relatives. Although all residents have a subsidized medication fee from the government, some caregivers face financial stresses as their income is limited. For example, Green explained how medication costs are expensive as some medications are costly than others. Green said that he is the eldest and his father is retired and his mother not working, so cost for medications and travelling to get his medications is costly which can reach 400 Qatari Riyals per month for a taxi excluding the medications. Others like Karkar explained that traveling with her mother for treatment outside the country is difficult to handle.

The non-Qatari retired old people face financial difficulties because they do not have pension but as long as they are residents they have subsidized medication fees only. But as Green mentioned above, one income is not sufficient and cost of medications and taxis are expensive which makes it financially hard for the caregivers. In addition, formal assistance from the government is not available which makes it even harder for some caregivers as they have to pay from their own income. 


\section{Discussion}

It seems that when caregiving roles are well established they can become taken-for-granted. We suggest that there is a serious under recognition of the amount of care provided to ageing relatives that may jeopardize the quality of life for both the elderly and their family caregivers. We suggest this has important policy implications. In Qatar currently, while there is generous state funded healthcare, the formal, state supported social structures for supporting elderly care are not yet well established and integrated. Within the concerns regarding adequate support of housebound elderly there is data to suggest that much of the burden for caregiving falls into the unpaid or poorly paid caregiving labour of women. The challenges that arise within providing care to a house or bed-bound older person is a particular challenge for poor and working class families who are unable to purchase formal care. The situation seems harder for families who are long term (often multi-generational) Qatari residents. They do not get any formal assistance from the government beyond subsidized medications. Although better off, organized home support for the Qatar nationals also has limitations.

It is our impression that cultural gender norms play a role in the experiences of family caregivers. Men are often viewed as being responsible for the financial security of the household. Caregiving is seen as primary and natural for women. Although several of the women primary caregivers we interviewed were employed outside the home, their income did not seem to be the main household income. These culturally accepted gender roles seem to justify men's limited involvement in the practical demands of care. This was particularly evident for both Green and May whose brothers did not contribute significantly to the caregiving. International studies into home caregiving for the elderly found that even when proximity is equal, sons are more likely than daughters to be distant from the practical, social and emotional aspects of taking care of their elderly parents (Walker et al, 1995:403). We noted that the daughters who are residing with their elderly family members in the same house described spending a great deal of each day helping and assisting them with their activities. There was also a thread in the data that indicated that the elderly person expected a high level of commitment from their daughters - perhaps more than from their sons who, even when identified as the primary caregiver for a highly dependent elder, seemed to have more leniencies to pursue interests outside the home. For example, Blue, who, despite being the primary caregiver for his bedridden, severely disabled father also attended university full-time.

The interview data from the participants who responded to our research recruitment revealed data that caregivers face many challenges in the caregiving process. In fact, the caregiving process caused burden and stress to the caregivers. As it was seen from the previous paragraphs, for example Bless checking the environmental quality for her elderly person, or Hope organizing and planning time management, or Mony in providing emotional needs or Blue, caring for his bedridden father but also, the dedicated work of the young male nursing student Mo who was primary caregiver for his severely disabled father and the other cases such as Noor and May. All these challenges make the caregiving process a difficult task which can have in the long run an impact on the quality of the caregiving provided and the health and quality of life of the caregiver.

\section{Conclusion and Recommendations}

The home caregivers in this paper expressed loving concern for their elderly people. They seemed clear in the values, roles and responsibilities that compelled them to ensure that the elderly people for whom they provided care were comfortable, respected, and that health decline was minimalized as much as possible. They exerted a great effort and energy in their care taking and described situations when their own health and strength waned, the times that led to stress and burden. Thus, we recommend that formal support groups are needed to assist home caregivers. For instance health care providers should be tasked with implementing family support groups where health care providers could act as consultants for the varied issues family caregivers encounter. As well, caregivers could be taught specialized skills for working with the cognitive, hearing, visual, bladder and bowel issues that may accompany advanced age. These resources would support family caregivers to problem solve and enhance their communication skills. Facilitating support groups in Qatar would require insight into cultural and ethnic differences. Routine home care visits focused on building rapport and creating a space in which to discuss problems, successes and feelings regarding care giving would be welcomed by many of the caregivers in our study. A support group complemented by home visits would also provide a practical resource and address caregivers' feeling of being overwhelmed with the physical demands of care. Psycho educational skills should be available to those caregivers who show serious psychological strain. These supports could build caregiver insight to support their caregiving role. It could also support caregivers to deal with change, manage competing responsibilities and temper feelings of resentment, hopelessness and loss.

\section{Acknowledgement}

This paper was part of a project on family home caregivers for elderly people in Qatar. This project was funded by the Doha International Family Institute member of Qatar Foundation in Doha-Qatar.

Disclaimer: The paper reflects the personal opinion of the author and is not necessarily endorsed by the Doha International Family Institute. 


\section{References}

Bowers, B. J. (1987). Intergenerational Caregiving: Adult Caregivers and Their Aging Parents. Advances in Nursing Science, 9, 20-31.https://doi.org/10.1097/00012272-198701000-00006

Brody, E. M. (1990). Women in the middle: Their parent-care years. Springer Publishing Company, New York.

Butcher, H. K., \& Buckwalter, K. C. (2002). Exasperations as blessings: Meaning-Making and the caregiving experience. Journal of Aging and Identity, 7(2), 113-132. https://doi.org/10.1023/A:1015439218276

Cantor, M. H. (1983). Strain among caregivers: A study of experienced in the United States. The Gerontologist, 23, 597-604. https://doi.org/10.1093/geront/23.6.597

Capezuti, E., Deanne, Z., Mathy, M., \& Terry, F. (2008). Evidence Based Geriatric Nursing Protocols for best Practise. Available online http://www.nursingip.eu/cinema/EBN\%20geriatric.pdf (accessed 20 November 2015)

Dahlberg, L., \& McKee, K. (2014). Correlates of social and emotional loneliness in older people: Evidence from an English community study. Aging and Mental Health, 18(4). 504-514. https://doi.org/10.1080/13607863.2013.856863

Dwyer, J. W., \& Coward, R. T. (1991). A Multivariate Comparison of the Involvement of Adult Sons versus Daughters in Care of Impaired Parents. Journal of Gerontology: Social Sciences, 46, 5259-5267. https://doi.org/10.1093/geronj/46.5.s259

Economic and Social Commission for Western Asia (ESCWA). (2013). Ageing in the Arab region: Trends, implications and policy options. Available online:

http://www.escwa.un.org/divisions/div_editor/Download.asp?table_name=divisions_other\&field_name=ID\&FileI $\mathrm{D}=1588$ (accessed 13 April 2015).

Given, B., Sherwoon, P., \& Given, C. (2008). “a”. What knowledge and skills do caregivers need? Journal of Social Work Education, 108(9), 115-123. https://doi.org/10.5175/JSWE.2008.773247703

Given, B. A., Given, C. W., \& Kozachik, S. (2001). 'b”. Family Support in Advanced Cancer. CA: A Cancer Journalfor Clinicians, 51, 213-231. https://doi.org/10.3322/canjclin.51.4.213

Grunfeld, E., \& Ian, M. (1997). Caring for Elderly People at Home: The Consequences to Caregivers. Canadian Medical Association Journal, 157(8), 1101-1105.

Hawkley, L. C., \& Cacioppo, J. T. (2010). Loneliness matters: A theoretical and empirical review of consequences and mechanisms. Annals of Behavioural Medicine, 40, 218-227. https://doi.org/10.1007/s12160-010-9210-8

Lin, I., Holly, F., \& Hsueh-Sheng, W. (2012). Negative and Positive Caregiving Experiences: A Closer Look at the Intersection of Gender and Relationship. Family Relations, 61(2), 343-358. https://doi.org/10.1111/j.1741-3729.2011.00692.x

Lou, Q., Shuling, L., Ya, H., Mengyuan, L., Shuai, L., \& Yong, J. (2015). Comprehensive Analysis of Patient and Caregiver Predictors for Caregiver Burden, Anxiety and Depression in Alzeheimer's Disease in Journal of Clinical Nursing, 24, 2668-2678. Available online: http://onlinelibrary.wiley.com/doi/10.1111/jocn.12870/pdf (accessed 20 June 2016).

Messecar, D. (2012). Nursing Standard of Practise Protocols. Available online: http://consultgeri.org/geriatric-topics/family-caregiving (accessed 15 May 2016).

Milligan, C. (2005). From home to 'home': situating emotions within the caregiving experience. Environment and Planning, 37, 2105-2120. https://doi.org/10.1068/a37419

Neal, M., Berit, I., \& Marjorie, S. (1997). Gender and Relationship Differences in Caregiving Patterns and Consequences Among Employed Caregivers. The Gerontological Society of America, 37(6), 804-816. https://doi.org/10.1093/geront/37.6.804

Northouse, L. L., Mood, D., Templin, T., Mellon, S., \& George, T. (2000). Couples' Patterns of Adjustment to Colon Cancer. Social Science Medicine, 50, 271-284. https://doi.org/10.1016/S0277-9536(99)00281-6

Phillips, L. R. (1989). Elder-family caregiver relationships: Determining appropriate nursing interventions. Nursing Clinics of North America, 24, 795-807.

Piercy, K. (1998). Theorizing about Family Caregiving: The Role of Responsibility. Journal of Marriage and Family, 60(1), 109-118. https://doi.org/10.2307/353445

Qatar statistics Authority. (2010). Economically active population (15 years and above) by nationality, sex \& sector . 
http://www.qix.gov.qa/discoverer/app/econnection?event=connectWithKey\&connectionKey=cf_a101\&clientType =viewer (accessed 8 February 2016)

Rudestam, K., \& Newton, R. (2001). Surviving your dissertation: A comprehensive guide to content and process. Sage Publications Inc: California.

Sandelowski, M. (2000). Focus on Research Methods. Whatever Happened to Qualitative Description. Research in Nursing and Health, 23, 334-340. https://doi.org/10.1002/1098-240X(200008)23:4<334::AID-NUR9>3.0.CO;2-G

Saxena, P. (2008). Ageing and age-structural transition in the Arab countries: regional variations, socioeconomic consequences and social security. Genus, 64, 37-74

Schoenfelder, D. E., Swanson, J. K., Pringle, S., Meridean, M., \& Johnson, M. (2000). Outcome indicators for direct and indirect caregiving. Clinical Nursing Research, 9, 47. https://doi.org/10.1177/10547730022158438

Schumacher, K., Barbara, S., Patricia, A, Mildred, C., Faith, M., \& Sangeeta, A. (2008). Effects of caregiving Demand, Mutuality, and preparedness on family caregiver outcomes during cancer treatment. Oncology Nursing Forum, 35(1). https://doi.org/10.1188/08.ONF.49-56

Singleton, J. (2000). Ethnicity and Gender in Non-Traditional Family Forms: Studies of Families Pushing Normative Boundaries. Journal of Comparative Family Studies, 31(3). 367-375.

Smith, C. E. (1994). A Model of Caregiving Effectiveness for Technologically Dependent Adults Residing at Home. Advances in Nursing Science, Dec 17(2), 27-40. https://doi.org/10.1097/00012272-199412000-00006

Stein, C., Virgina, W., Marcia, W., Michelle, G., Andrew, F., \& Thomas, J. (1998). "Because they are my parents": An Intergenerational Study of Felt Obligation and Parental Caregiving. Journal of Marriage and Family, 60(3), 611-622. https://doi.org/10.2307/353532

Swanson, E, Deborah, J, Janet, S, Marion, J., \& Meridean, M. (1997). "Caregiving: concept analysis and outcomes” in Scholarly inquiry for nursing practice: an international journal, 11(1), 65-75.

Teschendorf, B., \& Carolyn, S. (2007). Caregiver Role Stress: When Families Become Providers. Cancer, Culture and Literacy, 14(2), 183-189.

United Nations. (2012). World Population Prospects: The 2012 Revision, Available on: http://esa.un.org/unpd/wpp/unpp/panel_indicators.htm.

Walker A., Clara C., Pratt \& Lindy, E. (1995). Informal Caregiving to Aging Family Members: A Critical Review. Family Relations, 44(4), 402-411. https://doi.org/10.2307/584996

Work Family and Researcher Network. (2010). "Caregiving” available online: https://workfamily.sas.upenn.edu/glossary/c/caregiving-definitions (accessed 20 June 2016).

\section{Copyrights}

Copyright for this article is retained by the author(s), with first publication rights granted to the journal.

This is an open-access article distributed under the terms and conditions of the Creative Commons Attribution license which permits unrestricted use, distribution, and reproduction in any medium, provided the original work is properly cited. 